\section{Energiesysteme im Wandel}

\author{
von Wolfram Krewitt, DLR Stuttgart
}

Welche Rolle spielt "Life Cycle Assessment" (LCA) bei der Bewertung der Optionen, die uns dem Ziel einer nachhaltigen Energieversorgung näher bringen? Eine richtungssichere Langfriststrategie lässt sich wohl auch ohne LCA identifizieren, da Klimaschutz und zur Neige gehende fossile Ressourcen keine Alternativen zur Erhöhung der Energieeffizienz und der verstärkten Nutzung erneuerbarer Energien zulassen. Beispiele aus dem Bereich der Biomassenutzung zeigen, welch wichtigen Beitrag LCA bei der Ausgestaltung konkreter Handlungsoptionen liefern kann. Es wird außerdem zum methodischen Experimentieren eingeladen, um LCA auch in der prospektiven Technikbewertung sinnvoll einsetzen zu können.

\section{LCA als Kompass im Transformations- prozess?}

Die historisch gewachsenen Strukturen der Energieversorgung, die der westlichen Welt im letzten Jahrhundert scheinbar unbegrenzte Möglichkeiten eröffnet und einen schnell wachsenden Wohlstand beschert haben, stellen uns nun vor Probleme, deren Reichweite der größte Teil der Gesellschaft erst zu spät erkannt hat:

- der übermäßige Verbrauch begrenzter fossiler Rohstoffe, der inzwischen zu spürbaren wirtschaftlichen, sozialen und auch geopolitischen Verwerfungen führt;

- die sich abzeichnende globale Klimaveränderung. Die Industrieländer sind für rund 90 Prozent der bis heute durch den Energieeinsatz entstandenen $\mathrm{CO}_{2}$-Emissionen verantwortlich. Das starke wirtschaftliche Wachstum der Schwellenländer führt zu weiteren deutlichen Anstiegen;

- die extrem großen Unterschiede im Energieverbrauch zwischen Industrie- und Entwicklungsländern. In den ärmsten Ländern der Welt muss die Bevölkerung pro Kopf mit einem Dreißigstel der Energie eines Nordamerikaners auskommen. Nach wie vor haben rund 2 Mrd. Menschen auf der Erde keinen Zugang zu elektrischer Energie, was wieder- um entsprechende Auswirkungen auf die Bildungs- und Entwicklungschancen hat;

- die Risiken der Kernenergienutzung durch die Möglichkeit eines Kernschmelzunfalls, durch die notwendige Behandlung und dauerhafte Lagerung von Abfallstoffen und durch Proliferation. Die gegenwärtige IranKrise zeigt, wie schnell ein Konflikt um die Atomenergienutzung die Weltgemeinschaft an die Schwelle einer militärischen Auseinandersetzung führen kann.

Wäre alles anders gekommen, wenn LCA nur früher erfunden worden wäre? Wohl kaum, muss man sich eingestehen. Diese Einsicht mag jedoch hilfreich sein, wenn es darum geht, die Erwartungen an das Instrument Lebenszyklusanalyse nicht unangemessen hoch zu schrauben. Brauchen wir LCA, um der Politik zur Bewältigung der genannten Probleme eine richtungssichere Strategie zu empfehlen, die uns den Zielen einer nachhaltigen Energieversorgung näher bringt? Auch diese Frage ist wohl eher zu verneinen, denn bei zur Neige gehenden fossilen Ressourcen, der Notwendigkeit der $\mathrm{CO}_{2}$-Reduktion und den Risiken der Kernenergienutzung gibt es keine Alternativen zur Erhöhung der Energieeffizienz und der verstärkten Nutzung erneuerbarer Energien. Wie überaus wichtig aber der Gedanke der Lebensweganalyse bei der Ableitung konkreter Handlungsempfehlungen ist, bekommt man spätestens dann vor Augen geführt, wenn eine deutsche EEG-Förderung von Strom aus Biomasse zu Importen von Palmöl aus Malaysia führt und der Umwelt möglicherweise einen Bärendienst erweist. Die analytisch spannende Frage lautet damit: Welche Rolle kann das Instrument der Lebenszyklusanalyse spielen, um Nachhaltigkeitsdefizite in unserem Energiesystem zu erkennen und Lösungswege aufzuzeigen?

\section{LCA in der energiewirtschaftlichen Systemanalyse}

Im Bereich der Energiewirtschaft ist das Instrument LCA für industrielle Akteure von untergeordneter Bedeutung geblieben. Die Entscheidung eines Investors für den Bau eines Kohleoder Gaskraftwerks oder für die Investition in einen Windpark oder in ein Kernkraftwerk wird nicht von den Ergebnissen eines Life Cycle 
Assessments beeinflusst. Im Gegensatz dazu gehört in der Wissenschaft LCA zu den etablierten Instrumenten der energiewirtschaftlichen Systemanalyse und Technikbewertung. Unter dem Eindruck der ersten Ölkrise wurde bereits in den 1970er Jahren damit begonnen, unter Berücksichtigung des gesamten Lebensweges den kumulierten Energiebedarf verschiedener Technologien zu ermitteln, um deren Rolle in einem zukünftigen Energiesystem bewerten zu können. Die vergleichende Bewertung einzelner Technologien zur Strom- und Wärmeerzeugung ist dann auch das wesentliche Anwendungsfeld von LCA im Energiebereich geworden. Leider geriet dabei das Instrument der Lebenszyklusanalyse schnell zwischen die Fronten verschiedener energiepolitischer Interessen. Mit Berufung auf DIN- und ISO-Normen wurde mit LCA-Studien die Vorteilhaftigkeit von nahezu jeder Energietechnologie belegt. Als Blüte sei an die aus dem kumulierten Energieaufwand abgeleitete ,energetische Amortisationszeit" erinnert, die auch für ein Kohle- oder Kernkraftwerk, das immer mehr erschöpfliche Brennstoffe verbraucht als es Nutzenergie erzeugt, von manchen Autoren hartnäckig mit wenigen Monaten angegeben wurde (s. z. B. Voß 2005). Inzwischen wird akzeptiert, dass LCA nicht das ge- eignete Instrument ist, um die Frage nach der gesellschaftlichen Akzeptanz der Kernenergie oder der Windenergie zu beantworten. Die Bewertung der Risiken eines auslegungsüberschreitenden Kernkraftwerksunfalls liegt ebenso jenseits der methodischen Grenzen des LCAAnsatzes wie die Bewertung der Beeinträchtigung des Landschaftsbildes durch eine Windmühle auf der Schwäbischen Alb.

Von großem Nutzen war und ist das Instrument LCA immer dann, wenn es darum geht, verschiedene Technologien in einem ähnlichen Entscheidungskontext miteinander $\mathrm{zu}$ vergleichen. Interessante und energiepolitisch sehr relevante LCA-Studien gab es in den letzten Jahren zum Beispiel aus dem Bereich der Biomassenutzung. Die Biomassenutzung ist sowohl auf der Angebotsseite (Reststoffe und verschiedene Formen der Anbaubiomasse), auf der Nachfrageseite (Stromerzeugung, Wärmeerzeugung, Kraftstoffe) und auch bei den technischen Optionen der Energieumwandlung durch eine fast unüberschaubare Bandbreite verschiedener Optionen gekennzeichnet. Ohne eine methodisch saubere Lebenszyklusanalyse ist es kaum möglich, die Vor- und Nachteile der vielen Alternativen transparent aufzuzeigen und für Entscheidungsprozesse konsistent aufzubereiten.

\section{Abb. 1: $\mathrm{CO}_{2}$-Minderungspotenzial durch den Einsatz von Restholz*}

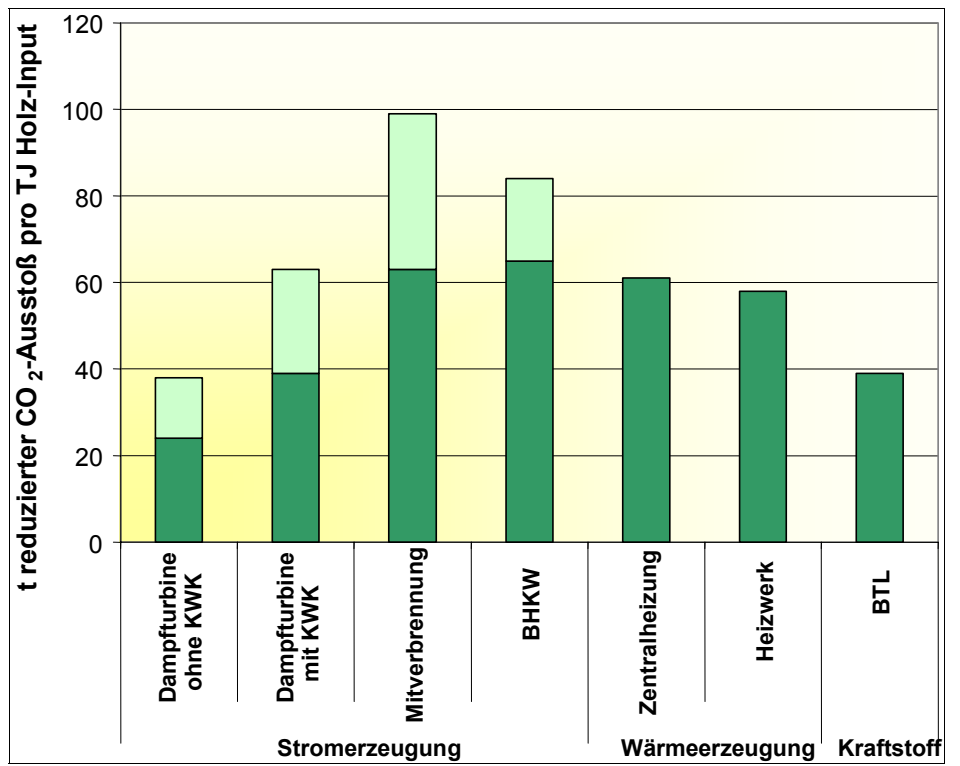

* Der helle Bereich in den ersten vier Balken zeigt die Bandbreite, abhängig von der jeweiligen Wärmegutschrift.

Quelle: Ramesohl et al. 2006 
Abb. 2: Nettoentlastung des Treibhauseffektes durch Biogasnutzung mit Gülle- und NawaRo-Anlagen*

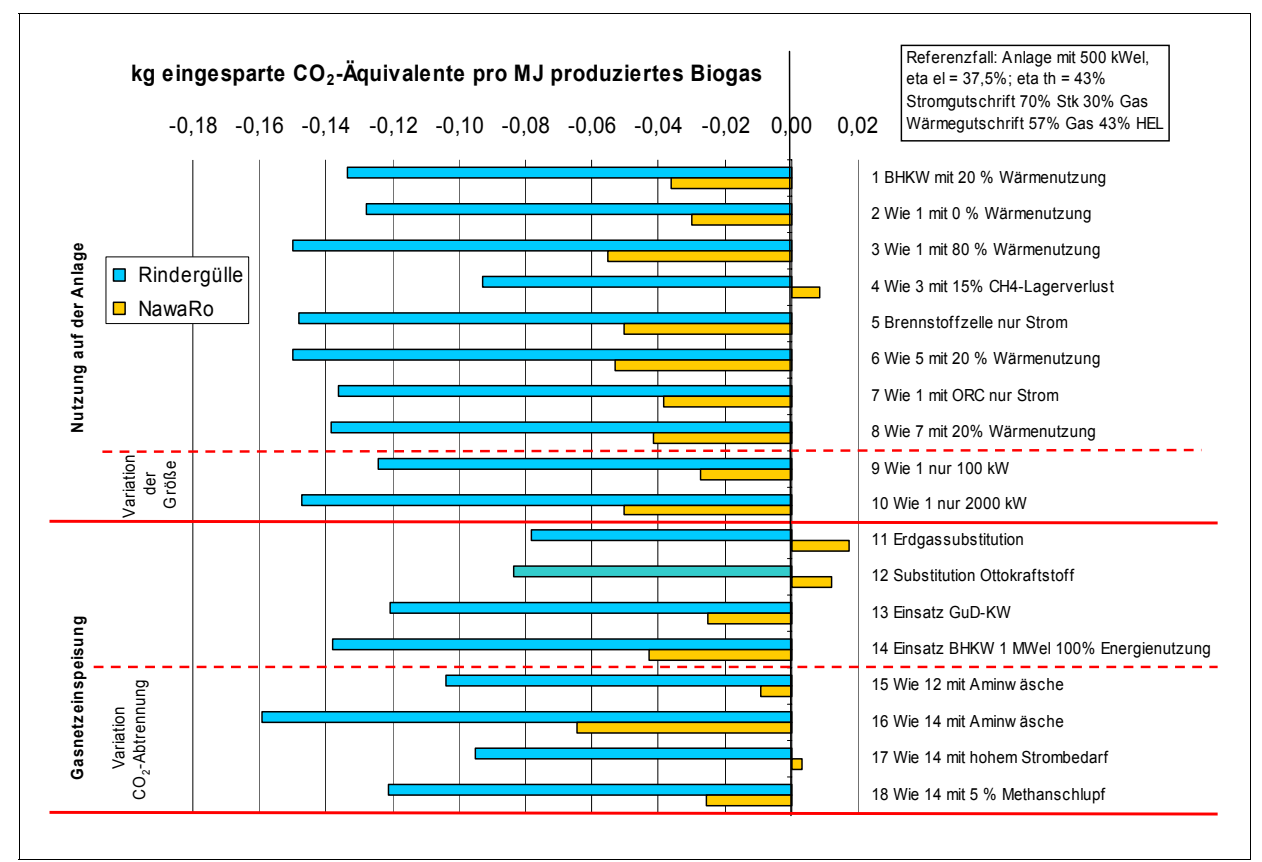

* NawaRo ist das Akronym für Nachwachsende Rohstoffe

Quelle: Pehnt 2007

Beispielhaft zeigt Abbildung 1 das $\mathrm{CO}_{2}$-Minderungspotenzial durch den Einsatz von Restholz in verschiedenen Anwendungsbereichen (Ramesohl et al. 2006). Es wird deutlich, dass unter Klimaschutzaspekten der Einsatz von Restholz in stationären Anlagen zur Strom- (in KraftWärme-Kopplung) und Wärmeerzeugung der Herstellung von Kraftstoff (BtL: Biomass-toLiquid) eindeutig vorzuziehen ist - ein Ergebnis, das durchaus im Widerspruch zu den ehrgeizigen politischen Zielen bei der forcierten Einführung von Biokraftstoffen steht. Als weiteres Beispiel aus dem Bereich der Biomassenutzung zeigt Abbildung 2 die Nettoentlastung des Treibhauseffekts durch die Nutzung von Biogas aus Gülle und Nachwachsenden Rohstoffen (NawaRo) (Pehnt 2007). In den meisten Fällen führt die Biogasnutzung zwar zu einer deutlichen Reduktion von Treibhausgas-Emissionen, die Entlastung kann aber je nach Rahmenbedingungen und eingesetzter Technologie durchaus unterschiedlich ausfallen. Im Einzelfall, wie bei der Aufbereitung von Biogas aus nachwachsenden Rohstoffen auf Erdgasqualität und Einspeisung in das Erdgasnetz oder beim Einsatz von Biomethan aus NawaRo als Kraftstoff kann es aber unter Berücksichtigung der Prozesse des gesamten Lebenszyklus auch zu einer Erhöhung der Treibhausgasemissionen kommen. Für die Gestaltung politischer Rahmenbedingungen zur Förderung der Biomassenutzung sind die in detaillierten Lebenszyklusanalysen gewonnenen Aussagen von höchster Relevanz. Die Politik kann sich glücklich schätzen, dass die wissenschaftliche Politikberatung in diesem Kontext auf eine etablierte und weitgehend standardisierte Methodik zurückgreifen kann.

\section{Mit LCA in die Zukunft sehen?}

Auf größere Schwierigkeiten stößt jedoch der Versuch, den LCA-Ansatz in der prospektiven Bewertung zukünftiger Technologieoptionen anzuwenden. In der Energiewirtschaft liegen Investitionszyklen im Bereich von 30 bis 50 Jahren; eine heute getroffene Investitionsentscheidung kann Strukturen für das nächste halbe Jahrhundert festlegen. Strategische Entscheidungen im Energiesektor haben also besonders langfristige Auswirkungen. Die Instrumente, die unter diesen Randbedingungen zur Entscheidungsfindung herangezogen werden, müssen in der Lage sein, die zeitliche 
Dynamik langfristiger Entwicklungen adäquat abzubilden - hier tut sich die Lebenszyklusanalyse eindeutig schwer. Methodisch korrekt zeigen verschiedene LCA-Studien aus den 1990er Jahren, dass die über den Lebensweg kumulierten $\mathrm{CO}_{2}$-Emissionen der Stromerzeugung aus Photovoltaik (damaliger Stand der Technik) in der gleichen Größenordnung wie die Emissionen eines erdgasgefeuerten GuD-Kraftwerks liegen. Welche Bedeutung hat eine solche Aussage im Hinblick auf die Ausrichtung einer langfristigen Forschungs- und Technologieförderung? Zum Teil ist auf der Grundlage solcher Ergebnisse die These aufgestellt worden, dass die Photovoltaik in der Zukunft keinen Beitrag zum Klimaschutz leisten kann. Eine statische Interpretation von LCA-Ergebnissen kann damit innovationshemmend wirken.

Um LCA im Rahmen der prospektiven Technikbewertung sinnvoll einsetzen zu können, müssen langfristige Entwicklungsperspektiven berücksichtigt werden. Hier gibt es vielfältige Wechselwirkungen zwischen sozioökonomischen Rahmenbedingungen, technischen Entwicklungsmöglichkeiten, der Ausgestaltung des zukünftigen Energieversorgungssystems und dem Life Cycle Inventory einer einzelnen Technologie (s. Abb. 3). Die damit verbundenen Unsicherheiten stehen zum Teil durchaus im Konflikt mit den in Normen festgeschriebenen Anforderungen an Lebenszyklusanalysen.

Im Rahmen des EU-Projekts „New Energy Externalities Developments for Sustainability“" (NEEDS), einem Integrated Project mit fast 70 Partnern aus ganz Europa, wird zur Zeit versucht, die komplexen Wechselwirkungen zwischen sozioökonomischen Rahmenbedingungen, den sich ändernden Strukturen des Energieversorgungssystems und den Entwicklungsmöglichkeiten einzelner Stromerzeugungstechnologien abzubilden (http://www.needs-project.org). Dies geschieht, um in einer, ,dynamischen Lebenszyklusanalyse“ Aussagen über die potenziellen Umweltbelastungen durch eine Technologie in der fernen Zukunft machen zu können.

Ausgehend von der Analyse eines technischen Systems (Photovoltaik, Brennstoffzelle, etc.) werden in NEEDS zunächst die Schlüsselprozesse identifiziert, die zur Optimierung der Funktion des Systems und zur Reduktion der Kosten führen. Die „Treibergrößen“, die diese Entwicklungen befördern oder hemmen (z. B. stringente Klimaschutzziele, gesellschaftliche Akzeptanz, staatliche F\&E-Aufwendungen), werden dabei beschrieben. In verschiedenen Diffusionsszenarien werden dann für jede einzelne Technologie mögliche technische Entwicklungspfade unter verschiedenen gesellschaftlichen und energiepolitischen Rahmenbedingungen aufgezeigt. Als Ergebnis dieser Analyse steht für jede Technologie die Charakterisierung technischer Konfigurationen zu unterschiedlichen Zeitpunkten und für unterschiedliche Entwicklungsszenarien zur Verfügung. Auf dieser Basis kann für verschiedene mögliche Konfigurationen einer zukünftigen Energietechnologie ein „Life-Cycle-Inventar“ ermittelt werden. Als Ergebnis steht in NEEDS nicht wie in LCA-Studien üblich ein einzelner Datensatz je Technologie zur Verfügung, sondern sämtliche LCA-Daten werden als Matrix für verschiedene Zeitpunkte (heute, 2025, 2050) und für unterschiedliche Entwicklungsszenarien aufbereitet.

\section{Abb. 3: Schema für die zu berücksichtigende Dynamik von Wechselwirkungen bei der Nutzung von LCA in der prospek- tiven Technikbewertung}

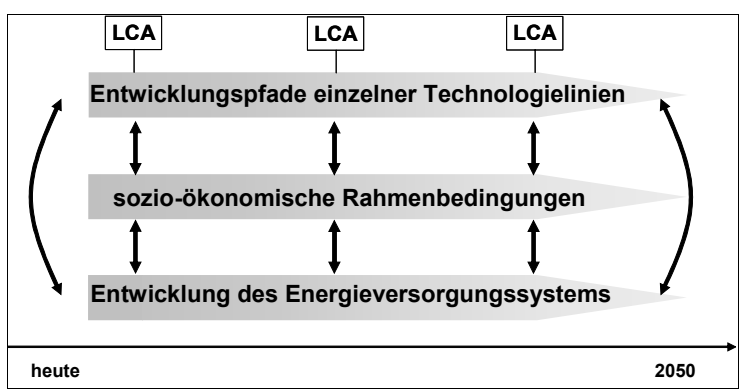

Quelle: Eigene Darstellung

Beispielhaft für einen möglichen „Technology Development Pathway“ zeigt Abbildung 4 die erwartete Entwicklung der Marktanteile verschiedener Photovoltaiktechnologien (Frankl et al. 2007). In einem ,optimistic-realistic scenario“, in dem unterstellt wird, dass die Förderung der Photovoltaik weitergeführt wird und damit ausreichende Anreize für technische Innovationen und ein weiterhin dynamisches Marktwachstum sicherstellt, wird erwartet, dass mittelfristig Dünnschichtzellen auf Kosten von Zellen aus kristallinem Silizium einen deutlich wachsenden Marktanteil erreichen, und dass bis 2050 sowohl konzentrierende Zellen mit hohem Wirkungsgrad als auch kostengünstige Poly- 
Abb. 4: Entwicklung der Marktanteile verschiedener Photovoltaik-Technologien in verschiedenen Szenarien

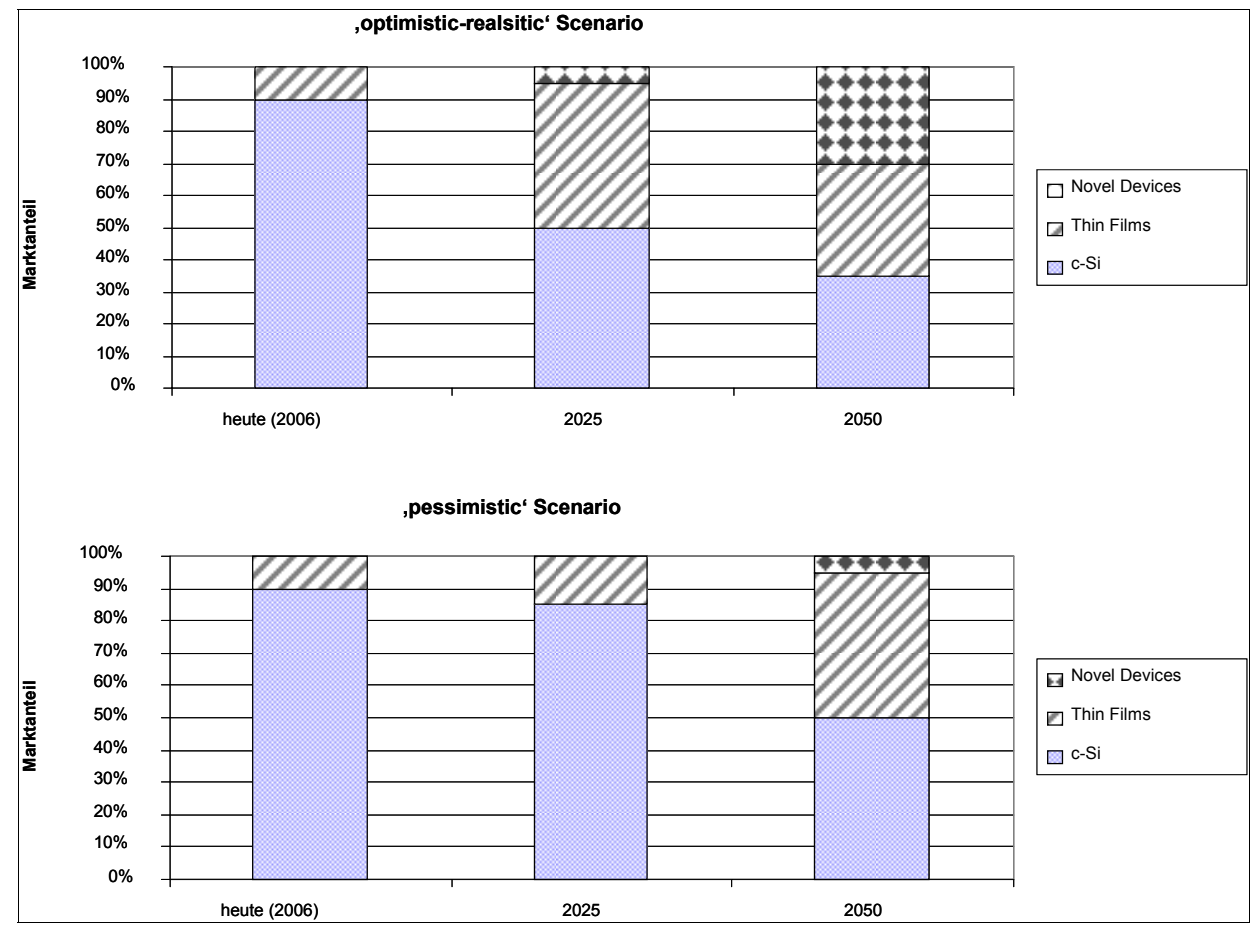

Quelle: Frankl et al. 2007

merzellen (beide hier als „novel devices“ zusammengefasst) etwa ein Drittel des Marktes abdecken. Wird dagegen die heutige Förderung der Photovoltaik nicht so lang durchgehalten, bis die Photovoltaik zumindest auf relevanten Teilmärkten wirtschaftlich konkurrenzfähig ist (,pessimistic scenario“), dann dürften die novel devices auch langfristig keine große Rolle auf dem Photovoltaik-Markt spielen.

Als Beispiel für die darauf aufbauenden LCA-Ergebnisse zeigt Abbildung 5 für die PVSchlüsseltechnologien in einem ,optimisticrealistic scenario" die kumulierten $\mathrm{CO}_{2}$-Emissionen je kWh an einem Standort in Südeuropa $\mathrm{zu}$ verschiedenen Zeitpunkten. Während die $\mathrm{CO}_{2}$-Emissionen für eine Mitte der 1990er Jahre produzierte und in Deutschland (Einstrahlung ca. $900 \mathrm{kWh} /\left(\mathrm{m}^{2}\right.$, a)) betriebene Dachanlage bei ca. $230 \mathrm{~g} / \mathrm{kWh}$ lagen (Marheineke 2002), liegen die Emissionen einer heute produzierten und in Südeuropa betriebenen Anlage nach Frankl et al. (2007) nur noch bei ca. $30 \mathrm{~g} / \mathrm{kWh} .{ }^{1}$ Unter Berücksichtigung weiterer technischer Entwicklungen - die auch notwendig sind, um die angestrebte Kostensenkung zu realisieren - ist zu erwarten, dass die $\mathrm{CO}_{2}$-Emissionen langfristig deutlich unter $10 \mathrm{~g} / \mathrm{kWh}$ sinken. Die Aufwendungen zur Herstellung konzentrierender Zellen mit sehr hohem Wirkungsgrad sind mit einer leichten Erhöhung der $\mathrm{CO}_{2}$-Emissionen verbunden.

Die Ergebnisse zeigen, dass das Potenzial zur Vermeidung von Treibhausgasemissionen durch die PV-Nutzung in einem zukünftigen Energiesystem außer Frage steht. Sollen LCAErgebnisse für strategische Fragestellungen verwendet werden, so ist es unbedingt notwendig, die zeitliche Dynamik zukünftiger Entwicklungen zu erfassen. Ohne die Berücksichtigung dieser Dynamik besteht die Gefahr, dass LCA-Ergebnisse bei der Bewertung zukünftiger Langfriststrategien kontraproduktiv wirken, da die Möglichkeiten innovativer Entwicklungen systematisch nicht abgebildet werden. 
Abb. 5: Kumulierte $\mathrm{CO}_{2}$-Lebenswegemissionen für verschiedene heutige und zukünftige Photovoltaik-Technologien*

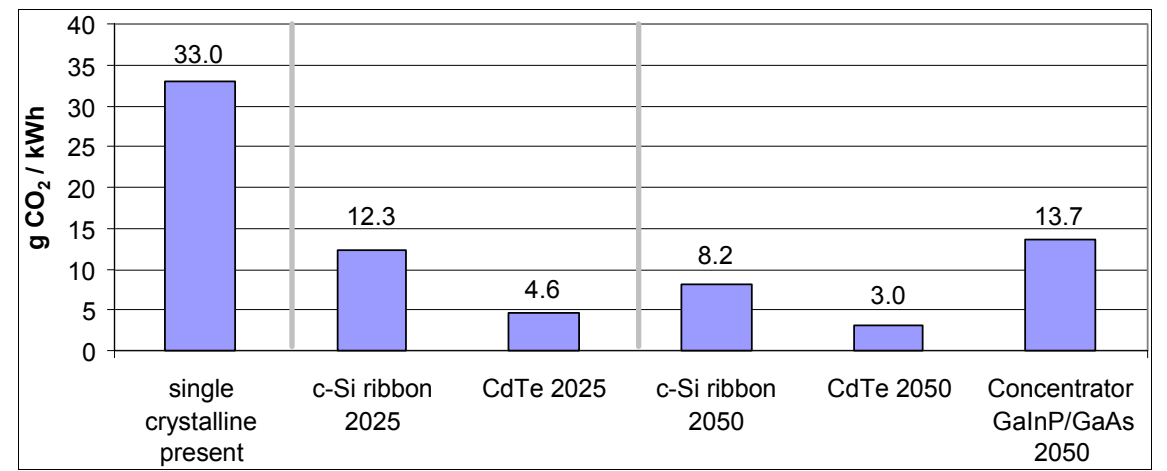

* Randbedingungen: Standort in Südeuropa mit $1800 \mathrm{kWh} /\left(\mathrm{m}^{2} \mathrm{a}\right)$, geneigtes Dach in Südausrichtung.

Quelle: Frankl et al. 2007

\section{Ausblick}

Einer der wesentlichen Treiber für den Transformationsprozess der Energieversorgung ist der Klimaschutz. Mit der Etablierung des europäischen $\mathrm{CO}_{2}$-Emissionshandels ist ein wichtiger Schritt zur institutionellen Verankerung der Klimaschutzmechanismen gemacht worden. Durch die Reduktion der zur Verfügung stehenden $\mathrm{CO}_{2}$-Zertifikate und deren zumindest teilweisen Versteigerung in der im nächsten Jahr beginnenden zweiten Handelsperiode wird die Wirksamkeit des Instruments deutlich erhöht. Verschiedene US-Bundesstaaten bereiten zurzeit die Einführung eines eigenen $\mathrm{CO}_{2}$-Handelssystems vor. Zum Teil wird dabei auch die transatlantische Vernetzung mit dem europäischen Handelssystem in Erwägung gezogen. Dies wäre ein entscheidender Schritt hin zu einem globalen Handel mit $\mathrm{CO}_{2}$-Emissionsrechten.

Im heutigen europäischen Handelssystem werden nur die direkten Emissionen der großen Emittenten (Kraftwerke, bestimmte Industriesektoren) berücksichtigt. Dies ist unbefriedigend, da Kleinverbraucher wie Haushalte und Verkehr, die zu einem wesentlichen Teil der Gesamtemissionen beitragen, nicht eingebunden werden. Dies führt dazu, dass die über den Lebensweg kumulierten indirekten Emissionen eines Produkts nicht verursachergerecht zugeordnet werden. Im Prinzip kann mit dem Instrument der Lebenszyklusanalyse für jedes Produkt die komplette $\mathrm{CO}_{2}$-Bilanz erstellt werden. Die Nutzung eines solchen Ansatzes in einem erweiterten $\mathrm{CO}_{2}$-Handelssystem ist aber zu kompliziert und nicht realisierbar. Viel ele- ganter wäre es, den Zertifikatehandel von der Ebene der Emissionen auf den „Upstream“ zu verlagern, also auf den Eintrag von kohlenstoffhaltigen Energieträgern in das Wirtschaftssystem. Mit diesem Ansatz, der z. B. zurzeit in Kalifornien diskutiert wird, könnten relativ einfach sämtliche Verbraucher fossiler Energieträger in das Handelssystem einbezogen werden.

Mit dem erfolgreichen Erneuerbare-Energien-Gesetz wurde in Deutschland ein klares Bekenntnis zur energie- und technologiepolitischen Bedeutung neuer Technologien zur Nutzung erneuerbarer Energien abgegeben. Durch die differenzierte Förderung der verschiedenen Technologien wurden stabile Rahmenbedingungen geschaffen, um die technischen, ökonomischen und ökologischen Entwicklungspotenziale der verschiedenen Technologien zu erschließen. Bei der konkreten Ausgestaltung und Bewertung alternativer Handlungsoptionen wird LCA weiterhin eine unverzichtbare Rolle spielen. Beispielhaft sei noch einmal die Diskussion um die Vor- und Nachteile der Aufarbeitung und Einspeisung von Biogas in das Erdgasnetz erwähnt. $\mathrm{Ob}$ es unter Klimagesichtspunkten Sinn macht, in einem Regenerativen-Wärme-Gesetz die Nutzung von Bio-Erdgas in einem Brennwertkessel als Alternative zur Installation einer Pellet-Heizung oder eines Solarkollektors zuzulassen, lässt sich nur mit einer transparenten Lebenszyklusanalyse bewerten.

Im Energiebereich wird das Instrument LCA weiterhin vor allem in der wissenschaftlichen Politikberatung seinen Platz haben. Die standardisierte Methodik bietet dafür eine hilf- 
reiche Grundlage. Wissenschaftliche Fragestellungen werden sich jedoch auch immer jenseits der durch Normen und Standards notwendigen Vorgaben bewegen. Ein vorsichtiges Experimentieren mit den methodischen Ansätzen wird nötig sein, um den Wandel des Energiesystems mit Augenmaß begleiten zu können.

\section{Anmerkung}

1) Um die Zahlen aus den verschiedenen Studien vergleichbar zu machen, kann in erster Näherung vereinfachend davon ausgegangen werden, dass die solare Strahlung in etwa linear in die Ergebnisse eingeht, d.h. die in Abbildung 5 dargestellten $\mathrm{CO}_{2}$-Emissionen wären an einem Standort in Deutschland etwa doppelt so hoch.

\section{Literatur}

Frankl, P.; Lombardelli, S.; Raugei, M., 2007: Life Cycle Impacts of Present and Future Photovoltaic Systems. Milan, $22^{\text {nd }}$ EU-PVSEC, 4 September 2007

Marheineke, T., 2002: Lebenszyklusanalyse fossiler, nuklearer und regenerativer Stromerzeugungstechniken. Forschungsbericht des Instituts für Energiewirtschaft und Rationelle Energieanwendung, Band 87, Universität Stuttgart

Pehnt, M., 2007: Biomasse und Effizienz. Vorschläge zur Erhöhung der Energieeffizienz von §8und §7-Anlagen im Erneuerbare-Energien-Gesetz. IFEU Institut, Heidelberg, Mai 2007; http://www. erneuerbare-energien.de/inhalt/39780/20049/

Ramesohl, S. et al., 2006: Entwicklung einer Gesamtstrategie zur Einführung alternativer Kraftstof$\mathrm{fe}$, insbesondere regenerativ erzeugten Wasserstoffs. Endbericht für das Umweltbundesamt, UFOPLAN FZK 20345118. Wuppertal Institut, DLR, IFEU. März 2006.

Voß, A., 2005: Nachhaltige Energieversorgung: Energietechniken auf dem Prüfstand. Ringvorlesung dipl.-ing. ikarus?, Stuttgart, 9. November 2005; http://www.ier.uni-stuttgart.de/publikationen/onpub av.html; download 14.12.2007

\section{Kontakt}

Dr. Wolfram Krewitt

Deutsches Zentrum für Luft- und Raumfahrt (DLR) „Systemanalyse und Technikbewertung“ am Institut für Technische Thermodynamik Pfaffenwaldring 38-40, 70569 Stuttgart

Tel.: +49 (0) 711 / 6862 - 766

E-Mail: wolfram.krewitt@dlr.de Internet: http://www.dlr.de 